\title{
Concept and Prelimnary Design of an Economical Bag Valve Mask Compressor as a Prototype for Simple Ventilator During COVID-19
}

\author{
Mubarak Muhamed Khan' ${ }^{1} \cdot$ Sapna Ramkrishna Parab $^{1}$ (D)
}

Received: 22 October 2020 / Accepted: 2 February 2021 / Published online: 11 February 2021

(C) Association of Otolaryngologists of India 2021

\begin{abstract}
The pandemic of COVID 19 has taken a massive toll of lives since its outbreak. Throughout the world with a large number of people being affected by covid 19, the need for the ventilators has risen. However, there is disproportionate ratio of demand versus supply of ventilators due to the menace caused by Covid 19 which has become unmanageable. This paper describes the design of the low cost portable mechanical bag valve mask compressor which could serve as a preliminary ventilator for the patients needing ventilator support in COVID 19. This prototype ventilator delivers breaths by compressing a conventional bag-valve mask (BVM) with a motor, eliminating the need for a human operator for the BVM. It is driven by a wind shield wiper electric motor powered by a $12 \mathrm{~V}$ battery. Additionally it can be used to deliver oxygen through either Laryngeal mask or compact face masks or nasopharyngeal airways where intubation is awaited in early breathlessness. Future additions for our prototype ventilator will include a controllable inspiration to expiration time ratio, a pressure relief valve, PEEP capabilities and an LCD screen. With a prototyping cost of only $\$ 150$, the concept of BVM compressor is a low-cost, low-power portable ventilator technology that will provide essential ventilator features at a fraction of the cost of existing technology.
\end{abstract}

Keywords COVID 19; SARS-CoV-2 · ARDS · Mechanical ventilator - Mortality; ventilator .

Sapna Ramkrishna Parab drsapnaparab@gmail.com

1 Sushrut ENT Hospital and Dr. Khan's ENT Research Center, Talegaon Dabhade, India
Bag valve mask (BVM) · Low-cost ·

Portable and automatic

\section{Introduction}

Coronavirus disease 2019 (COVID-19) is an infectious disease caused by severe acute respiratory syndrome coronavirus 2 (SARS-CoV-2). Common symptoms include fever, cough, and shortness of breath. While the majority of cases result in mild symptoms [1], some progress to pneumonia and multi-organ failure [2, 3]. As of 29th August 2020, the total number of Coronavirus cases worldwide are 24,943,292 with 842,014 deaths and 61,152 (1\%) serious or critical patients [4] with as many as about $10 \%$ of positive cases needing ventilation. Considering the total requirement for ventilators, the number of ventilators and assisted breathing devices required for the total number of patients are very less. This situation is present even in the affluent countries. There is a gross shortage of medical ventilators worldwide to treat such a large number of COVID 19 infected patients in developed as well as developing countries. Acute respiratory distress syndrome (ARDS) is diagnosed in those with severe illness resulting from coronavirus which is associated with a high mortality. ARDS, at times is initially diagnosed as pneumonia or pulmonary oedema. Patients with coexisting previous lung disease (asthma, COPD) may develop respiratory failure and mechanical ventilation and supportive therapies are the mainstays of treatment due to hypoxemia. [5] The severity of the disease is the primary indicator to individualise treatment. Ventilator support may be invasive or non-invasive. Invasive mechanical ventilation is via an endotracheal tube or tracheostomy with breaths delivered by a mechanical ventilator. It is recommended for patients with 
moderate or severe ARDS. Non-invasive ventilation is via mask or nasal prongs and is for patients with mild ARDS who is hemodynamically stable, is easily oxygenated, does not need immediate intubation, and has no contraindications to its use [6].

With the increase in mortality and morbidity associated with COVID 19, the demands for requirement of ventilator have increased. The disproportionate demand versus supply/ availability of ventilators have put a health burden on the hospitals by deprivation of the needy ARDS patients for ventilator support. Modern ventilators are computerized equipment, but patients can also be ventilated with a simple, hand-operated bag valve mask. In order to be effective, a bag valve mask must deliver between 500 and 800 milliliters of air to a normal male adult patient's lungs, but if supplemental oxygen is provided $400 \mathrm{ml}$ may still be adequate [7]. Squeezing the bag, 10-12 respirations per minute in an adult and 20 per minute in a child or infant provides an adequate respiratory rate [8]. We have designed a low-cost, simple, easy to use and easy to build mechanical Bag valve mask compressor that can serve to help the COVID patients who will be needing supportive breathing assistance. COVID 19 pandemic may continue in near future and demand of ventilators and simple economical breathing assisting devices might grow steadily.

We put forth a simple preliminary prototype ventilator model which is based on mechanical compression of BVM (Bag Valve Mask). It will be both inexpensive, cost effective as well as easily available to the needy ARDS patients and early breathless patients for non- invasive ventilation.

\section{Device Design}

The prototype design of our BVM compressor consists of

- Bag Valve Mask (BVM) / Air Delivery through any compressible self-inflating balloon

- Compressor piston for BVM

- Motor for creating piston movement

All these components when housed within a chamber serves as basic model of a primitive ventilator or breathing assisting device (Fig. 1).

\section{Air Delivery Technique}

The commercially available BVM works on the mechanism of manual compressions of the self-inflating bag. The effective transfer of oxygen into the lungs depends on the efficient compression of the BVM and the adequate seal of the mask onto the mouth of the patient. The operator

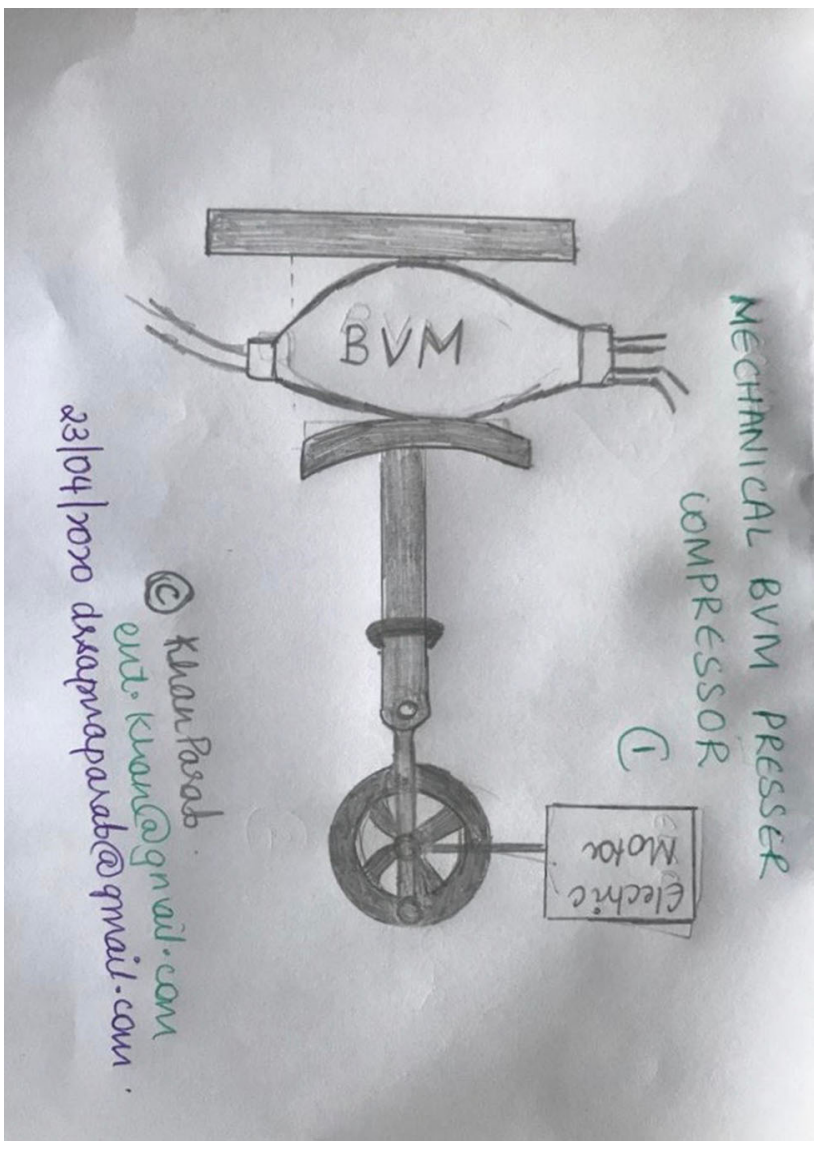

Fig. 1 First prototype of our BVM compressor based on to and fro push back concept

squeezes the bag as well as holds the mask on the patient. This may result into fatigue of the operator doing both the actions simultaneously. The efficiency may also be limited by the skill of the operator. Our methodology, therefore, was to design a motor driven mechanical compressor device to automatically compress the BVM at regular intervals. This prototype design of a compressor which will be cost effective and will serve the basic function of providing artificial respiration to the patient with ventilation dysfunction.

A bag valve mask (BVM), also known by proprietary name AMBU (Artificial Manual Breathing Unit) bag, or "self-inflating bag". The bag-valve mask concept was developed in 1953 by the German engineer Holger Hesse and his partner, Danish anaesthetist Henning Ruben [9].

The BVM consists of

1. Mask: The mask has to be properly sealed around the patient's face to deliver the right pressure to inflate the lungs. It is present in smaller sizes for paediatric patients. 2. Bag and valve: On deflation, it self-inflates from its distal part drawing in either ambient air or a low pressure oxygen flow from cylinder. Bag and valve combinations 
can also be attached to an endotracheal tube or laryngeal mask airway. When attached to oxygen cylinder it delivers nearly $100 \%$ [10].

\section{First Prototype Based on to and Fro Push Back Concept of our BVM Compressor}

It consists of:
1. BVM
2. Convex plank
3. Piston
4. Connecting rod
5. Wheel
6. Motor

The BVM is compressed by the piston action of the rod attached to the convex plank in contact with the BVM. The piston is attached to the connecting rod to a wheel whose motion will drive the piston into to and fro action compressing the BVM. All these actions are motorised. The motor is attached to a wheel (Fig. 2).

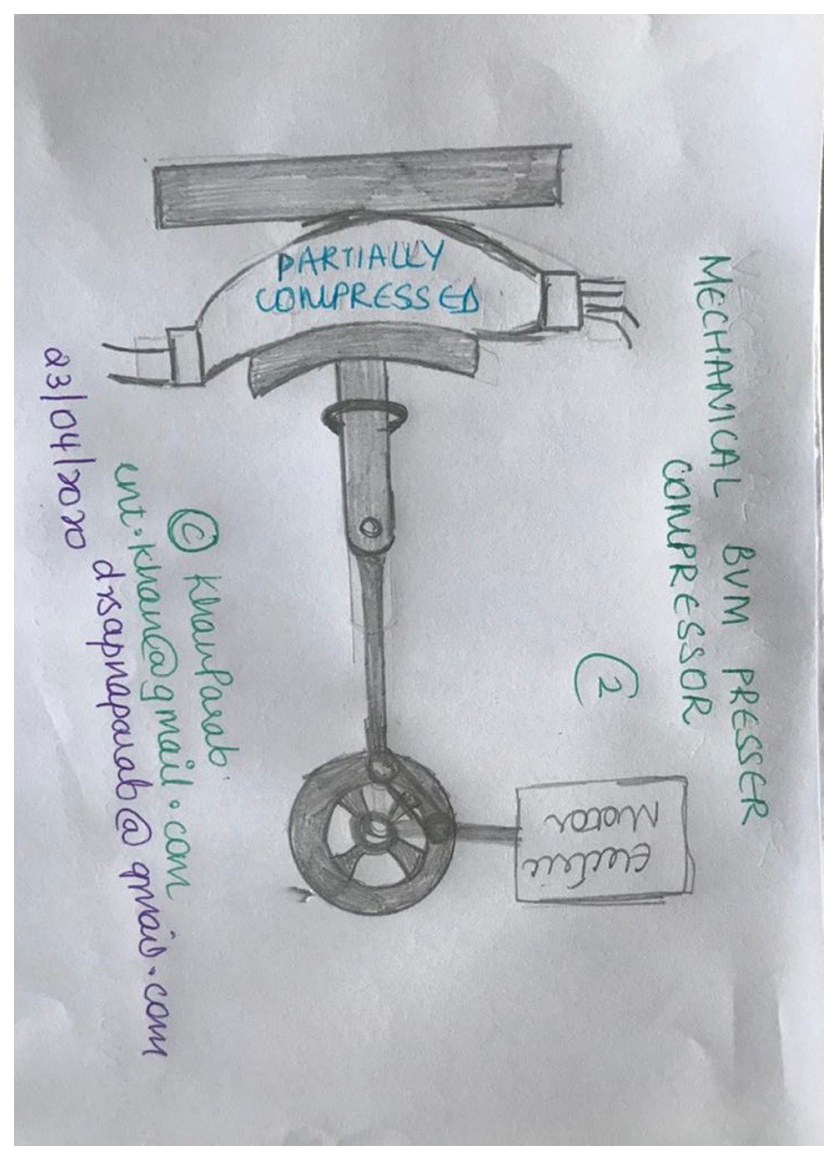

Fig. 2 Compressed state of first prototype of our BVM compressor based on to and fro push back concept
Alternate Model for Mechanical Bag Valve Mask Compression

\section{A. Rack and piston mechanical Compression}

It consists of

1. BVM

2. Convex plank

3. Piston

4. Crank pin with slotted arm

5. Rotating disk

On rotating the disk carrying the crank pin working in the slotted arm, reciprocating (to and fro) rectilinear motion is imparted to the rack at the bottom by the vibration of the toothed sector (Fig. 3).

\section{Wind Shield Wiper Motorised Action of Mechanical Compression}

Our BVM compressor is driven by motor. We use the motor used for Wind shield wiper whose speed can be

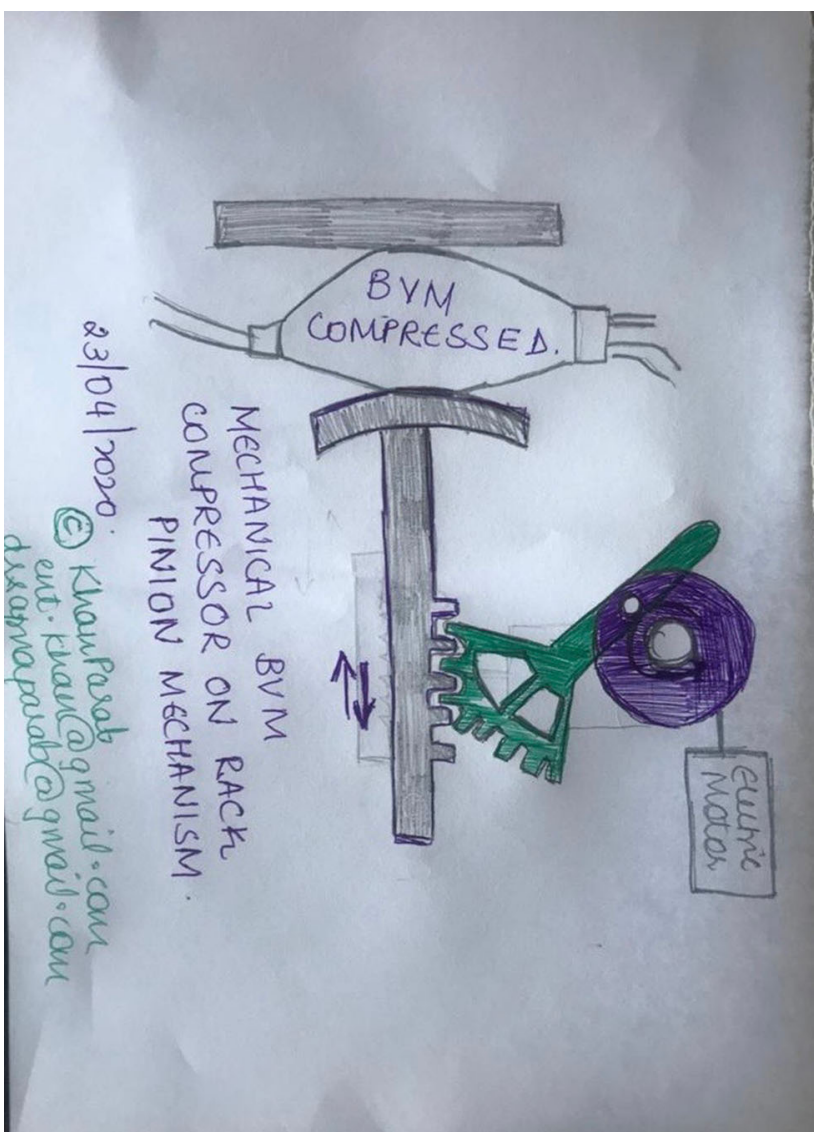

Fig. 3 Alternate model for mechanical Bag Valve Mask compression 
controlled with rotating switch. Wiper motors are devices in the wiper system that functions on a power supply in order to move the wiper blades in a smooth motion. Like other motors, the wiper motor rotates continuously in one direction which is converted into a back and forth motion. As the wind shield wiper motor [11] allows pre-decided, rhythmical actuated movements of the wiper, similarly this mechanism allows predetermined number of compression of the bag valve mask. The compression rate can be altered and adjusted by increasing diameter of wheel on rotating arm of motor. A windshield wiper is a device used to remove rain, snow, ice, and debris from a vehicle's front window so the vehicle's operator can better see what's ahead of them. The motor in the middle converts the circular rotation to an intermittent rotation. The wiper motor is a DC (direct current) $12 \mathrm{~V}$ motor that includes a set of gears and a park switch. The park switch allows the motor to stop when wipers are positioned at the bottom of the windshield.

\section{The Actual Idea and Working}

This simple prototypes of our BVM compressor is based on the motorised mechanical compressions which can be adjusted so as to deliver 12 compressions in one minute for an adult and 20 compressions in a minute for a paediatric patient. This will deliver oxygen into the lungs of the patient by controlling the motor rotations through adjustable rotating switch. On compression of the AMBU bag, the oxygen inside the bag is force-fed into the lungs of the patient via a one-way valve into the patient's trachea. In intubated/ tracheostomised patient, the mechanical compressor tubing is directly connected to the endotracheal tube/ tracheostomy tube. In case of non-intubated patient with respiratory dysfunction, either mask or laryngeal or nasopharyngeal airways are used. The masks will be provided in different sizes to fit infants, children, and adults. The masks will be disposable. At present there are no pressure calibrations onto our ventilator, but these can be added to precisely know and monitor the pressure of the oxygen pumped.

Future Modifications for the Mechanical BVM

1. Filters between the mask and the bag.

2. Positive end-expiratory pressure (PEEP) valve.

3. LCD Monitor with display of pressures.

4. Pressure relief valves.

\section{Advantages}

1. Ventilation support during Mass casualty such as corona pandemics, natural disasters and massive toxic chemical releases

2. During transport of intubated patients through ambulance to higher medical centres

3. In primary rural hospitals as primary aid in ventilation

4. Breathing support in non-invasive ventilation

5. Basic primary life supportive device in road traffic accidents and ambulances

6. Basic ventilator onsite in comatose patients in high risk professions

7. In small set up for basic ventilation for minor operative procedures

8. In mining, chemical industries etc, such portable ventilator is of vital importance to tackle ventilation problems arising out of occupational hazards.

9. User friendly, cost effective and portable

10. When incorporated with pressure valves, inspiration to expiration time ratio, a pressure relief valve, PEEP capabilities and an LCD screen, it can function as a ventilator proper.

11. It can be used to deliver oxygen through either Laryngeal mask or compact face masks or nasopharyngeal airways where intubation is awaited in early breathlessness

12. When housed within a chamber it will be portable ventilator.

\section{Strengths}

Simple prototype of ventilator based on day-to-day technique of life support

\section{Limitations}

At present although it's a concept and idea, it can be subjected to AUTOCAD software and can be easily manufactured with cost effective measures for perfect portability.

\section{Conclusion}

This concept and the design can be developed into a basic prototype of a simple ventilator which will serve minimal function of tiding over the crisis of transportation of patient from smaller center to the one with good ventilator support. 
Acknowledgement We express our gratitude to Dr. Shirin Khan for the technical support provided and to Asim Khan for the proof reading of the manuscript.

Author Contributions MMK: Instrument design and development, manuscript drafting, editing SRP: Instrument development, manuscript drafting, editing.

Funding This study was not financially supported from external sources.

\section{Compliance with Ethical Standards}

Conflict of interest The design has been applied to Design Act, India under the name of Dr Mubarak Khan.

\section{References}

1. Gu Jinyang, Han Bing, Wang Jian (2020) COVID-19: Gastrointestinal manifestations and potential fecal-oral transmission. Gastroenterology. https://doi.org/10.1053/j.gastro.2020.02.054.ISSN00165085.PMID32142785

2. Hui DS, I Azhar E, Madani DA, Ntoumi F, Kock R, Dar O et al (2020) The continuing 2019-nCoV epidemic threat of novel coronaviruses to global health-The latest 2019 novel coronavirus outbreak in Wuhan, China. Int $\mathrm{J}$ Infect Dis 91:264-66. https://doi.org/10.1016/j.ijid.2020.01.009.PMID31953166

3. "Q\&A on coronaviruses". World Health Organization (WHO). Archived from the original on 20 January 2020. Retrieved 27 January 2020.

4. https://www.worldometers.info/coronavirus/

5. Budweiser S, Jörres RA, Pfeifer M (2008) Treatment of respiratory failure in COPD. Int J Chron Obstr Pulm Dis 3(4):605

6. Chatburn RL, Mireles-Cabodevila E (2013) Chapter 3 basic principles of ventilator design. In: Tobin MJ (ed) Principles and practice of mechanical ventilation. McGraw-Hill, New York

7. Daniel Limmer and Michael F. O'Keefe (2005) Emergency Care 10th ed. Edward T. Dickinson, Ed. Pearson, Prentice Hall. Upper Saddle River: New Jersey

8. https://www.ahajournals.org/doi/full/ https://doi.org/10.1161/circ.102.suppl_1.1-22

9. Ambu's history“. Ambu Ltd. Archived from the original on 2011-04-27

10. Stoy, Walt (2004). Mosby's EMT-Basic Textbook (PDF). Mosby/JEMS. ISBN 978-0-323-03438-8

11.

https://ru.boschautomotive.com/en/parts_and_accessories/ electronics_and_accessories/electric_motors/wiper_motors/ wiper_motors_1

Publisher's Note Springer Nature remains neutral with regard to jurisdictional claims in published maps and institutional affiliations. 